\title{
Setenta Años de contribuciones de la Escuela Agrícola Panamericana a la Protección de los Suelos
}

\author{
Gloria Arévalo $^{1}$ y Carlos Gauggel $^{2}$
}

\begin{abstract}
Resumen. La unidad de suelos de la Escuela Agrícola Panamericana, Zamorano, Honduras, ha participado desde su fundación en la formación de profesionales con excelencia académica y un conocimiento profundo y práctico de los principios de suelos y aguas, que contribuya substancialmente al incremento de la producción de alimentos, pero conservando y mejorando el medio ambiente. En el transcurso del tiempo, ha evolucionado a ser una unidad que cumple con su principal objetivo de formar profesionales y se proyecta a brindar apoyo técnico al sector agropecuario, desde pequeños productores a empresas multinacionales y entes gubernamentales, como unidad de servicio y proyección de la institución. La unidad de suelos se ha posicionado en uno de los pilares de la agricultura sostenible al usar la tecnología más avanzada, poseer un equipo técnico altamente capacitado y la participación activa de los estudiantes en todas las actividades técnicas y docentes en las que se ocupa. El Laboratorio de Suelos hace parte de la unidad de suelos y en el se realizan análisis químicos de suelos, aguas y tejido vegetal, que se ha proyectado como uno de los más confiables como respaldo de la actividad agropecuaria de los países de la región. Además, implementó una parcela demostrativa para prácticas de conservación de suelos y un club de suelos como actividades académicas y de extensión.
\end{abstract}

Palabras clave: Agricultura sostenible, conservación de suelos, club de suelos, laboratorio de suelos.

\section{Panamerican Agriculture University, Zamorano, has Contributed during Seventy Years in Soil Protection}

\begin{abstract}
The Soils Unit of the Panamerican Agriculture School, Zamorano, Honduras, has been involved since its foundation, in professional training of students with academic excellence and a deep and practical knowledge of soil and water management, contributing substantially to increasing food production responsibly with the environment. The unit has kept to its main objective to produce graduates capable of facing and solving problems in the soil sciences. The Soils Unit does outreach to provide technical support to the agricultural sector, from small producers to multinational corporations, government agencies and other outreach units. The Soils Unit is positioned as one of the pillars of sustainable agriculture using the latest technology, having a highly trained technical team and the active participation of students in all activities and teaching techniques of the unit. The Soils Unit includes the Laboratory for Chemical Analysis of soil, water and plant tissues, which ranks as one of the most reliable laboratories supporting farming activities in the regional countries. A soils conservation demonstration plot and a soils club for students are extension and academic activities coordinated by the Soils Unit.
\end{abstract}

Key words: Soil club, soil conservation practices, Soils Laboratory, sustainable agriculture.

\section{Introducción}

Los suelos han estado ligados a Zamorano (Escuela Agrícola Panamericana) desde el comienzo de la institución, ya que fue uno de los principales criterios de la selección del lugar donde estaría ubicada la Escuela Agrícola Panamericana. En un artículo que escribió para la inauguración de Zamorano, el Dr. Wilson Popenoe exclama "Aquí teníamos suficiente tierra, a una elevación apropiada para la mayoría de los cultivos tropicales" (Malo 1999).

Desde sus inicios, se ha incluido en sus programas la enseñanza de suelos, ya que Zamorano es una universidad que ha venido evolucionando a través

\footnotetext{
${ }^{1}$ Profesor Asociado de Manejo de Suelos, M.Sc. Unidad de Suelos, Carrera de Ingeniería Agronómica, Escuela Agrícola Panamericana, Zamorano, Honduras, correo electrónico ggauggel@ zamorano.edu

${ }^{2}$ Profesor Adjunto, Ph.D. Unidad de Suelos, Carrera de Ingeniería Agronómica, Escuela Agrícola Panamericana, Zamorano, Honduras. correo electrónico carlos.gauggel@ hotmail.com
} 
del tiempo tanto organizativa como científicamente. Este dinamismo ha dado lugar a que las unidades que la componen ofrezcan ahora opciones y oportunidades en educación a los estudiantes y la actualización de los docentes para satisfacer las necesidades en los países de los cuales provienen nuestros estudiantes y prestan sus servicios una vez graduados.

Una de las unidades de la Carrera de Ingeniería Agronómica que ha mostrado este dinamismo de cambio ha sido la unidad de suelos. Esta ha cambiado de ser una sección meramente docente a ser una unidad docente, de investigación, extensión y servicios a la comunidad agrícola en Honduras, Centro América y algunos países de América del Sur. A partir de 1999, cuando se instauraron las cuatro carreras, los suelos se involucraron en la carrera de Desarrollo Socioeconómico y Ambiente, ampliando las aplicaciones de suelos no solamente a la producción agropecuaria, sino también a su influencia en el ambiente y el desarrollo de la población. Con las necesidades en el mundo de generar y adaptar tecnología y conocimiento a la protección del ambiente, a la disminución de emisiones de carbón y su captura, y a la disminución del impacto del cambio climático, desertificación, mejor uso y aprovechamiento del agua disponible para la agricultura y la vida humana, y la producción de bioenergía, se amplía la necesidad de aplicar el conocimiento básico de suelos a la resolución de estos temas, generando la oportunidad para docentes y alumnos de integrar el conocimiento de la ciencia del suelo a estos temas.

Estos cambios en el enfoque de la unidad de suelos han requerido la ampliación, actualización y adaptación de los programas académicos, de las prácticas de campo y del equipo analítico de última generación en el laboratorio de suelos, además, a formar y emplear profesionales dedicados a diferentes especialidades y aplicaciones en suelos.

\section{Reseña Histórica de la Unidad de Suelos}

La necesidad de la enseñanza de la ciencia del suelo en Zamorano fue reconocida desde su fundación y las clases de suelos han sido parte del programa académico de Zamorano. Los importantes criterios que W. Popenoe tuvo que satisfacer en la búsqueda del sitio adecuado eran: El tipo de suelo tenía que ser fértil, pero no mucho, un promedio para diferentes cultivos y con buen drenaje. Las opciones de lugares eran planicies ubicadas en los valles de Honduras como en Jamastrán, Danlí, Yoro, Comayagua, Quimistán, Talanga, Cedros, Santa Bárbara, El Espino, Siria, Yeguare, Olanchito, Sulaco y Valle de Ángeles (Malo 1999).

El valle de Quimistán había sido uno de los favoritos de W. Popenoe, especialmente porque tenía los suelos que a él le gustaban. Eran profundos con mucha ceniza volcánica reciente y con buen drenaje. Él y sus colegas estaban de acuerdo que los suelos con alta fertilidad no hubiera sido lo mejor para enseñar agricultura científica, lo cual fue uno de los factores que incidió en la selección del valle del Yeguare como uno de los que cumplía con esta condición de suelos de categoría limitada para la mejor producción agrícola (Malo 1999).

En su alto interés por la formación de jóvenes al servicio de la agricultura y extender las prácticas de producción de alimentos, W. Popenoe recibió informe del Sr S.Y Lin, de la FAO, en 1954 en un estudio sobre la posibilidad de la explotación ictiológica en Honduras. Para ello se reseñan los suelos de montañas de origen volcánico antiguo en la región central y sur del país, en las que pone de manifiesto la degradación de los suelos por erosión, su poca profundidad y la exposición de la roca como limitantes para su uso y la necesidad constante se aplicación de nutrientes y materia orgánica para mantener su productividad; y en los pequeños valles como el de Zamorano, la presencia de arcillas pesadas que limitan la calidad de los suelos (Lin 1954). Esta condición de los suelos también la exponen Monroe (1968), Yuksel y Robles (1979), Díaz Zelaya (1989) y se mantiene en la actualidad (Barahona Flores 2000, Chica et al. 2008, Dubón Fernández 2007, Velásquez et al. 2008), lo que exige un manejo cuidadoso y adaptado para lograr producir cultivos de calidad y competitivos en el mercado local e internacional. Ese es el escenario de suelos sobre el cual se fundó la Escuela Agrícola Panamericana y su esquema de aprendizaje en el manejo de los suelos para sus usos y aplicaciones.

El Dr. Daniel Meyer, Profesor de Acuacultura, reseña la actividad de la unidad de suelos en Zamorano desde los años 60’s bajo la dirección del 
español, Dr. Fernando Fernández de Córdova, como profesor de suelos, quien se graduó en Zamorano en 1958 y Ph.D. en suelos de la Universidad de Florida. El Dr. Fernández fue también Decano de Zamorano y a su salida de la institución, trabajó en CIAT y después fue Director de Fundación Hondureña de Investigación Agrícola (FHIA) en Honduras. Otro egresado Zamorano de la primera clase en 1946, Francisco Sierra, Ph.D. en suelos, también fungió como profesor de suelos a finales de los 60's y comienzos de los 70's. Por esta época existió una colección de rocas que todavía se usa para la enseñanza del curso básico de suelos. Los graduados de la clase 74 tuvieron como profesor de suelos al español Fernando Sierra, de la cooperación del gobierno español. En esa época, no había módulos, los estudiantes se rotaban de un profesor a otro ${ }^{3}$. Entre 1975 y 1977 fue profesor de suelos el Dr. Allan Haworth y de 1978 al 80 el profesor fue Dan Walters, quien organizó el Laboratorio de Suelos, ambos eran voluntarios del Cuerpo de Paz de los Estados Unidos de América.

El Dr. Hugh Popenoe, hijo del Dr. Wilson Popenoe y Fiduciario de Zamorano y Fiduciario Emérito hasta su muerte en el 2011, obtuvo su Ph.D. en suelos de la Universidad de Florida, y fue quien recomendó y llevó a Zamorano a la norteamericana, M.Sc. Nancy Erickson, como profesora de suelos en 1979. En los 90’s la Dra. Erickson obtuvo su título de Ph.D. en suelos en la Universidad de Florida. La historia de los suelos en Zamorano en la década de los 80’s y 90’s está ligada a la Dra. Erickson quien impartía las clases y laboratorios de química en primer año y Ciencia del Suelo en segundo.

En los 80’s, el laboratorio de química y suelos quedaba en el actual edificio de Recursos Humanos ${ }^{4}, \mathrm{y}$ fue durante la gestión de la Dra. Erickson, cuando se construyó el actual laboratorio de química en el sector de los edificios de Ciencias Básicas. Desde esa época, en el laboratorio de química se imparten las prácticas

\footnotetext{
${ }^{3}$ Pitty, A. 2012. Historia de catedráticos de suelos. Profesor Pleno. EAP, Zamorano, Honduras. Correo electrónico apitty@zamorano.edu).

${ }^{4}$ García, C. 2013. Remembranzas de la clase de suelos en los 80's. Profesor Pleno. EAP, Zamorano, Honduras (cgarcia@zamorano.edu).
}

de la clase de química a primer año y las de la clase de suelos en segundo año. La Dra. Erickson manejó este laboratorio con toda rigurosidad y celo, con sus asistentes, una de ellas la Ing. Química Griselda Montoya, quien laboró hasta 2004. Publicó los manuales de conferencias y laboratorio para la clase de suelos (Erickson 2001a, Erickson 2001b). La Dra. Erickson trabajó en Zamorano hasta el 2002, como profesora de química y Ciencia del Suelo, de casi 20 generaciones de Zamoranos.

En 1985, se realizó un seminario sobre fijación de nitrógeno, con la presencia del Profesor David Hubbel. Por esta época, se realizaron varias publicaciones acerca de la fijación de nitrógeno ${ }^{5}$. A finales de los 80`s, el laboratorio de suelos lo manejó el Ph.D. Marciano Rodríguez (panameño).

En convenio con la Dirección ejecutiva de Catastro de Honduras y Zamorano, se realizó el estudio de suelos a semidetalle del valle de Zamorano, como resultado del interés del jefe del Departamento de Agronomía de Zamorano y del Dr. Rodriguez, profesor de suelos (Díaz Zelaya 1989). Por Zamorano, participó en este estudio el recién graduado Agr. Oscar Díaz ${ }^{6}$. El Dr. Rodriguez, posterior a su retiro de Zamorano se fue a trabajar a San Pedro Sula en el norte de Honduras ${ }^{3}$.

En los 80's se vinculó a la actividad de suelos la entonces M.Sc. Ana Margot Andrews ${ }^{7}$. Comenzó su actividad montando el laboratorio de microbiología de suelos, con un proyecto de la Universidad de Florida. La Dra. Andrews realizó su tesis de doctorado en el laboratorio de suelos de Zamorano (Andrews 1990) y fue nombrada Directora del Laboratorio de Suelos en esta época. Impartía el tema de fertilidad de suelos, en la clase de suelos de segundo año que luego se convirtió en la clase de Fertilidad y Manejo de Suelos. En estos años, en la clase de tercer año impartía la clase de Nutrición Vegetal y participaba durante una semana en el tema de fertilizantes en la clase de

${ }^{5}$ Rosas, J.C. 2012. Historia del laboratorio de suelos. Profesor Pleno de EAP, Zamorano, Honduras. (Entrevista).

${ }^{6}$ Martinez, C. 2012. Historia del laboratorio de suelos Zamorano años 90. Instructora Jefe Técnico. EAP, Zamorano, Honduras. (Comunicación personal).

7 Andrews, A.M. 2012. Experiencias en Suelos en Zamorano, EAP, Honduras. Consultora independiente. Entrevista y correo electrónico. 
Agroquímicos que tenía a cargo el Ing. Mario Bustamante. Andrews también estaba a cargo de la supervisión de los módulos de suelos y del laboratorio de suelos, trabajando solo a medio tiempo; al final de su permanencia en Zamorano trabajaba $70 \%$ del tiempo. Durante esa época trabajó en mejorar la guía para el módulo de suelo, el trifolio de cómo tomar muestras de suelo y un informe sobre los daños causados por el huracán Mitch en Zamorano en 1998.También trabajó dentro del programa del frijol que dirige desde esa época el Dr. Juan Carlos Rosas ${ }^{5}$ en el tema suelos limitados por fósforo (Andrews et al. 1995, Andrews et al. 1994). Dirigió trabajos de tesis en las áreas relacionadas con el manejo y la conservación de suelos (Alpizar Vargas s.f., Ávila Cáceres 1991, Barahona Flores 2000, Barillas Lechuga s.f., Díaz s.f., Nuñez Servellón s.f., Paniagua et al. 1998, Pérez 1996, Pozo Gordillo s.f., Tamashiro 1997, Urrutia Tobar 2000), y publicó un resumen con las tesis en el área de suelos (Andrews 1993).

El Dr. Luis Caballero ${ }^{8}$ refiere que en los 90 's, con la Dra. Andrews, la unidad de suelos apoyó los programas del Departamento de Recursos Naturales y Conservación Biológica de la Escuela Agrícola Panamericana, en el manejo de cuencas hidrográficas y la conservación de suelos. La doctora Andrew se retiró de Zamorano en el 2002.

El Dr. Leonel Espinoza ${ }^{9}$, Ph.D. en suelos y graduado de Zamorano en 1982, refiere que las profesoras Erickson y Andrews fueron sus mentoras en suelos como estudiante en Zamorano y el mayor enfoque de los suelos se hacía en el manejo de la fertilidad. Él trabajó en el laboratorio de suelos en 1983 realizando análisis químicos.

El laboratorio era más o menos del mismo tamaño actual y ubicado en el mismo edificio (Edificio de Agronomía, Roberto García Carmona), pero en el ala contigua a donde está localizado actualmente. Con el apoyo del Dr. Jorge Chang, jefe de Agronomía hacia 1986, cuando enfocó su esfuerzo a la calibración y

${ }^{8}$ Caballero, L.A. 2012. Participación en el programa de suelos de la EAP Zamorano. Reminiscencias. Profesor asociado de EAP, Zamorano, Honduras. (Entrevista).

${ }^{9}$ Espinoza, L. 2012. Participación personal en el programa de suelos de Zamorano. Profesor Universidad de Arkansas, Estados Unidos. (Correo Electrónico) funcionamiento de los equipos instrumentales en el laboratorio, donde se hacían análisis básicos de suelos, usando la solución Mehlich 3 como extractante y determinación de materia orgánica con el método de Walkley-Black (Arévalo y Gauggel 2011). La actividad se concentraba en los años 80 en la parte académica y el servicio al público era limitado. Durante esta misma época, existió un acuerdo de colaboración entre la Escuela Agrícola Panamericana y un programa de manejo de cuencas bajo la dirección del Ministerio de Recursos Naturales.

Motivado por su padre, que era un extensionista especializado en suelos, el Dr. Espinoza había decidido desde joven obtener su doctorado en suelos, lo cual logró, desarrollando su carrera académica en universidades de los Estados Unidos, hasta obtener su Ph.D. Ejerce su carrera actualmente en la Universidad de Arkansas, desde donde sigue apoyando a Zamorano ubicando a jóvenes egresados en programas de esa universidad, con miras a especializarse en suelos.

En 1987, se vinculó al laboratorio de suelos como instructora la Ing. Química Hilda Flores, hondureña, quien realizaba los análisis químicos de suelos, aguas para riego, tejido vegetal, abonos y fertilizantes, velando por la precisión y el rigor de los análisis de suelos. Permaneció en el cargo durante 23 años hasta diciembre de 2010 cuando se retiró.

En 1988, se vinculó a Zamorano el Agr. Manuel Rosales, bajo la dirección del Ing. Nelson Agudelo ${ }^{10}$, en el programa forestal, hasta 1994 cuando falleció, aun estando al servicio de Zamorano. El Agr. Rosales había trabajado en el levantamiento de suelos de Honduras, con personal del catastro nacional, lo que lo hizo gran conocedor de la geología del país y lo aplicó a Zamorano en el manejo de carreteras en el Uyuca. También contribuyó en hacer una colección de rocas de la región, que quedó en manos del Ing. Agudelo y a su retiro en 2013 en las del Rector Dr. Roberto Cuevas García.

En 1992, llega desde el CIAT a Zamorano, el Dr. Silvio Viteri, microbiólogo colombiano, quien trabajó en los proyectos de producción de micorrizas y Rhizobium con el Dr. Juan Carlos Rosas. Viteri fue profesor del módulo de Conservación de Suelos y de

\footnotetext{
10 Agudelo, N. 2012. Manejo del programa forestal de la
} EAP Zamorano. Profesor Pleno, EAP, Zamorano. 
Microbiología de Suelos entre 1992 y 1996. Participó en el desarrollo de tesis relacionadas con el uso de abonos verdes para el mejoramiento del suelo (Vásquez Maldonado 1994) y la fijación de nitrógeno (Wong 1992) ${ }^{5}$.

En los años 80's y 90's hubo programas de financiamiento, refiere el Dr. Rosas. El auge por el trabajo en temas de regeneración de la calidad del suelo por medios naturales fue el efecto de la primera subida de petróleo en los 80's, lo que aún promueve la búsqueda de alternativas al uso de fertilizantes sintéticos bajo el esquema de la agricultura intensiva y evitar la degradación de los suelos.

En los 90's se vinculó al programa de conservación de suelos el entonces Ing. Luis Caballero $^{8}$ como instructor del módulo, junto con los ingenieros zamoranos J. Tamachiro y M. Vásquez ${ }^{6}$. Se realizaron ensayos de manejo de suelos en laderas, cultivos de cobertura, restauración de suelos, trabajando con leguminosas de cobertura como mucuna (Mucuna spp.) y Dolichos lablab y Leucaena (Leucaena leucocephala), con variedades provenientes desde México a Colombia, para producir medios forestales como leña e incorporar las hojas al suelo para su mejoramiento. Como parte del programa, se hacía extensión con los productores de la parte alta, media y baja de la cuenca de Güinope para la adaptación de la Leucaena a diferentes alturas. Los estudiantes del módulo salían de Zamorano para participar en los programas de extensión y realizar prácticas de suelos y aguas.

Se inició el proyecto centroamericano de Manejo Integrado de Suelos (MIS) y se logró promover la investigación en cuencas, financiando las primeras estructuras de flujos hidrológicos y vertederos que fueron utilizados para estudiar el manejo del control de pérdidas de suelo en cuencas hidrográficas en Siguatepeque y Güinope en Honduras, y en Nicaragua, dejando a varios productores altamente capacitados y fincas demostrativas en prácticas de conservación de suelos y aguas.

Con la ONG Vecinos Mundiales se trajeron instructores campesinos de Guatemala. Desde esa época, en Güinope existe un centro de investigación, experimentación y de aplicación de tecnologías. Parte de la financiación la generó la GTZ, cuando Raúl Zelaya era el director del programa de desarrollo rural del Yeguare. Con ello se financiaban los módulos de suelos y otros ${ }^{8}$. También se buscaron recursos económicos con programas de producción y venta de micorrizas y de yuca.

En la protección de cuencas se ha realizado una cadena de proyectos con la cual Zamorano ha ido creciendo y en los cuales la participación del equipo de suelos ha sido muy importante. Su aporte ha sido primordialmente en caracterización, diagnóstico y proyección del manejo de los suelos. Zamorano, a través de la unidad de Recursos Naturales y posterior carrera de Desarrollo Socioeconómico y Ambiente, ha participado en proyectos como en el PostMitch, 2001, en Nicaragua, MIRA de USAID hasta 2006, orientado al manejo de los recursos naturales en varios departamentos de Honduras. También participó en el proyecto UNID, financiado por la Fundación Kellogg Iniciativa del Yeguare, hasta el 2008, donde se manejaban suelos y cultivos ${ }^{8}$.

Proyectos para evaluar la adopción de tecnologías en el manejo de suelos para el control de cambio climático de suelos financiado por FAO 2011 y Propark 2012, enfocado al mejoramiento del uso de la tierra transformando la producción de cultivos anuales, altamente erosivos, en sistemas agro forestales con cobertura permanente y diagnóstico del suelo en áreas de amortiguamiento. La unidad de suelos ha sido clave, en la investigación del proceso hídrico, definiendo la aptitud de las clases de suelos, para utilizarlos en modelación ecológica con aplicación en proyectos en Honduras y otros países. También, en áreas cercanas a Zamorano y sus cuencas como Uyuca y Santa Inés, proveedoras de agua para la Escuela Agrícola Panamericana y las comunidades aledañas, se han realizado estudios y caracterización de suelos para generar modelos de manejo de cuencas, como es el caso de los estudios realizados por Martínez Martínez (2008), Lavaire Cruz y Fiallos (2010) y Acosta Velásquez y Kucharsky Lezama (2012). Estos estudios, además se convierten en escenarios de enseñanza y aprendizaje para estudiantes.

Zamorano se ha integrado al trabajo con la sociedad en el tema de manejo de suelos para protección de la cuenca hidrológica a largo plazo y en el mejoramiento socioeconómico para la comunidad y la sostenibilidad en la producción hídrica, integrado a 
la producción agropecuaria, disminución del impacto erosivo y degradativo del suelo, mediante la adopción de prácticas adecuadas en manejo de los suelos, integrado al manejo de cultivos, bosques y áreas protegidas.

En los años 90`s, Zamorano se vinculó al proyecto de Salud de suelo de universidades norteamericanas, principalmente la de Cornell. Se generaron manuales y metodologías de extensión rural para la aplicaciones prácticas de la ciencia de suelo, que luego adoptó el proyecto PROMIPAC para su gestión de extensión rural (Reilly et al. 2002).

En 1999, llega a Zamorano como profesor invitado el Dr. Erich Raddatz (Foto 1), científico alemán que trabajó muchos años en el aislamiento y producción de cepas seleccionadas de micorriza, con un enfoque al mejoramiento en la nutrición vegetal y aprovechamiento del agua en los cultivos. Se inicia entonces una época de investigación alrededor de este tema, dando a Zamorano la oportunidad de ofrecer tecnología biológica, natural y de mucho impacto en la producción vegetal. El tema continúa en desarrollo con aplicaciones en cultivos como café, palma aceitera, plátano, frutales y árboles maderables. Algunos de estos aportes se citan en la bibliografía (Andrade Yépez 2003, Cabanilla Burbano 2005, Calderón Díaz 2005, Canales Paguada et al. 2008, Chávez et al. 2006, Coello Wilches 2004, Crespo Mena 2006, Cruz Ortiz 2007, De La Cadena Vera 2005, Discua Duarte et al. 2008, Guerra González et al. 2009, Lagos Molina 2010, Lizama Meza y Vásquez Guillén 2001, Morales Rivera 2005, Patiño Mejía 2005, Posada et al. 2004, Romero Oseguera 2006, Segura Escobar 2010).

En el año 2001 se enseñaban dos cursos de suelos: uno a nivel introductorio en segundo año y otro avanzado enfocado en nutrición vegetal y fertilidad del suelo en tercer año de Ingeniería Agronómica. En Zamorano existen dos laboratorios de suelos, uno con fines netamente docentes y otro, analíticamente mejor equipado, para hacer trabajos de investigación como tesis y brindar servicios a terceros. Estos servicios eran limitados a pocos productores que hacían uso del servicio de análisis de suelos y recomendación de un programa de fertilización para un cultivo. El número de tesis en temas relacionados con suelos era poco y siempre relacionado con aplicación en la agricultura.

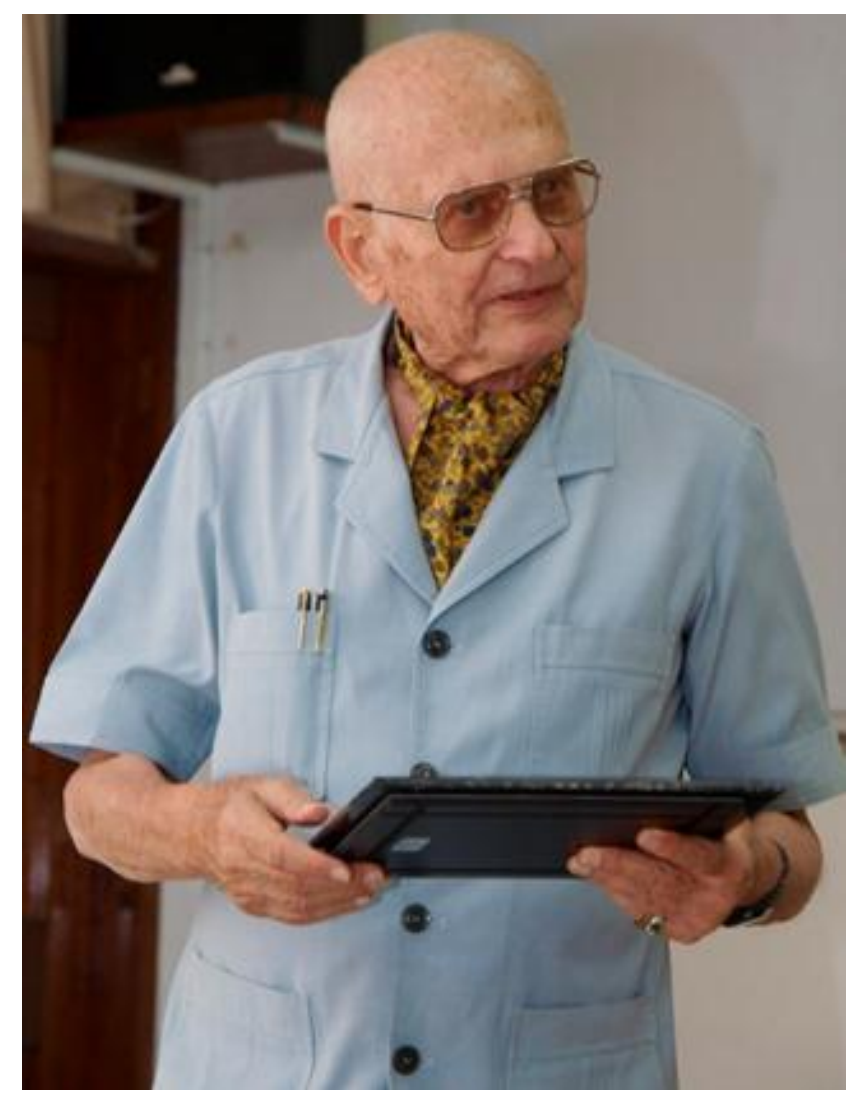

Foto 1. El Dr. Erich Raddatz recibiendo un reconocimiento otorgado por la Carrera de Ciencia y Producción Agropecuaria de Zamorano, Honduras, en el 2008.

En el 2002, se hace cargo de la unidad de Suelos el Dr. Carlos Gauggel, Ph.D. en suelos (Fotos 2 y 3), y se reconocen las oportunidades que un servicio integral de suelos que incluyera análisis de suelos, foliares y recomendaciones con su debido seguimiento al usuario, lo que traería al país y a la región grandes beneficios en la producción agrícola. De esta manera se reorganizó el laboratorio añadiendo al equipo profesional dos extensionistas para hacer promoción en Centroamerica, excepto Costa Rica. Creó un servicio de extensión y promoción de los servicios del laboratorio entre los productores. Esta gestión fue muy exitosa y resultó en posicionar al laboratorio de suelos de la Carrera de Ingeniería Agronómica entre los más utilizados por los productores del Norte de Centro América. 


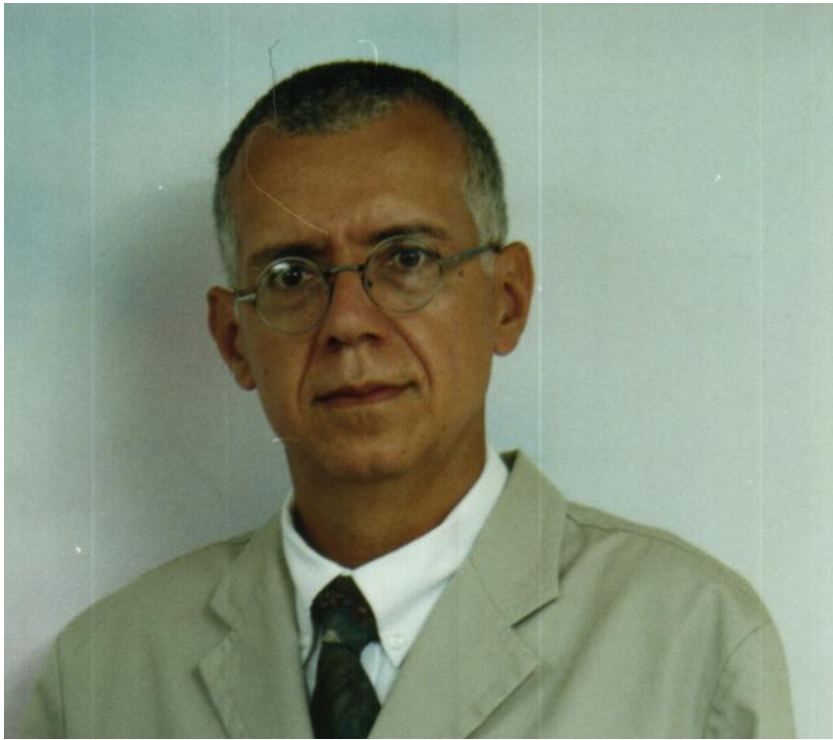

Foto 2. Dr. Carlos A. Gauggel, Director de la Unidad de Suelo de la Escuela Agrícola Panamericana, Zamorano, Honduras, de 2002 a 2004.

A partir del 2003, el laboratorio también ofreció los servicios de Estudios Agrológicos de Suelos (mapas de suelos para su uso), con recomendaciones para el manejo, adecuación, rehabilitación y conservación de los suelos mediante la aplicación de técnicas más adecuadas según la condición de los suelos, como métodos de labranza, diseño de drenajes, técnicas de conservación de suelos, más adecuadas según la condición de los suelos y dirigidos a la obtención de altos rendimientos en cultivos agrícolas con prácticas sostenibles. También realizó el primer mapa detallado de suelos de las áreas agrícolas de Zamorano, para ser utilizado en la programación de prácticas agrícolas en las que se toma en cuenta la calidad de los suelos y su potencial productivo, en los que participaron estudiantes de último año en la elaboración de sus tesis (Castro Vargas 2003, Fernández Díaz 2003, López Aguilar 2003, Terrones Cano 2003). Durante el periodo del Dr. Gauggel, varios estudiantes se motivaron a seguir especializaciones en el campo de suelos.

En 2003, los estudiantes de tercer año de la carrera de agronegocios, motivados por el curso básico de segundo año, solicitaron tomar el curso avanzado de tercer año. Este curso fue impartido con énfasis en el manejo de los recursos naturales, la industria de los fertilizantes y sus oportunidades, y el costo y beneficio del manejo sostenible del suelo. El Dr. Gauggel, continúa prestando su apoyo a la unidad de suelos, hasta la fecha de escribir este artículo, como Profesor Adjunto de Zamorano, vinculado a actividades de tesis, apoyo al laboratorio y a la academia.

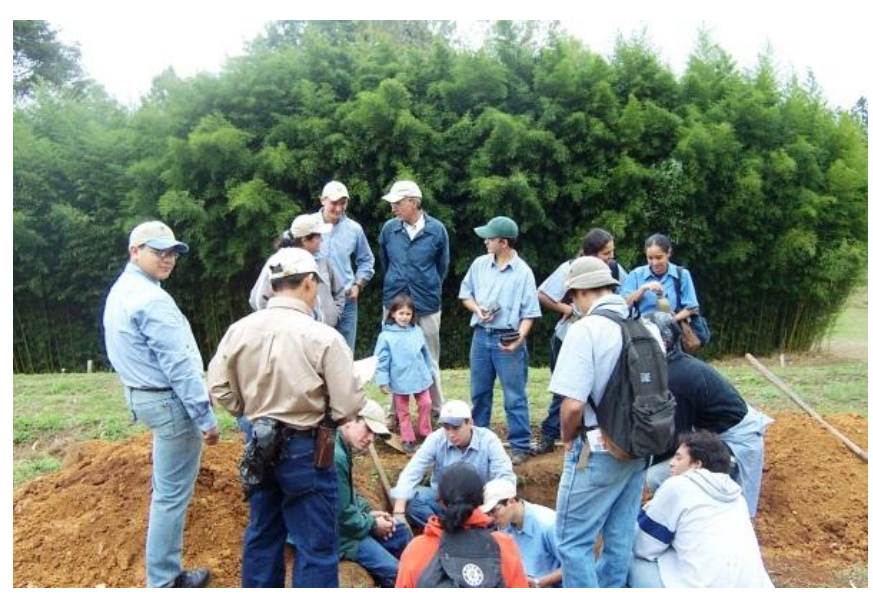

Foto 3. El Dr. Carlos A. Gauggel con un grupo de estudiantes en las cercanías del chalet Cabot en el Uyuca, en el 2003.

A partir de 2004 y hasta el 2013, la unidad de suelos ha sido manejada por la colombiana M.Sc. en Suelos y Aguas, Gloria Arévalo de Gauggel (Foto 4), imparte tres cursos de suelos diseñados dentro del programa académico: un curso básico de ciencia del suelo y aguas dirigida a los estudiantes de segundo año en el currículo general y la clase de Manejo de Suelos y Nutrición Vegetal en tercer año de la carrera de Ingeniería Agronómica. También imparte, en la Carrera de Ingeniería en Desarrollo Socioeconómico y Ambiente (antes DSEA, hoy IAD), el mismo curso de Manejo de Suelos y Nutrición Vegetal, que reconociendo la necesidad y la gran importancia del medio ambiente y el papel que el suelo juega en ello, se fue modificando hasta plantearse en un nuevo curso de Manejo y Conservación de Suelos, que enfatiza en la restauración de la calidad del suelo, conservación del suelo y transferencia de tecnología mediante el uso de varios métodos de clasificación de suelos. 


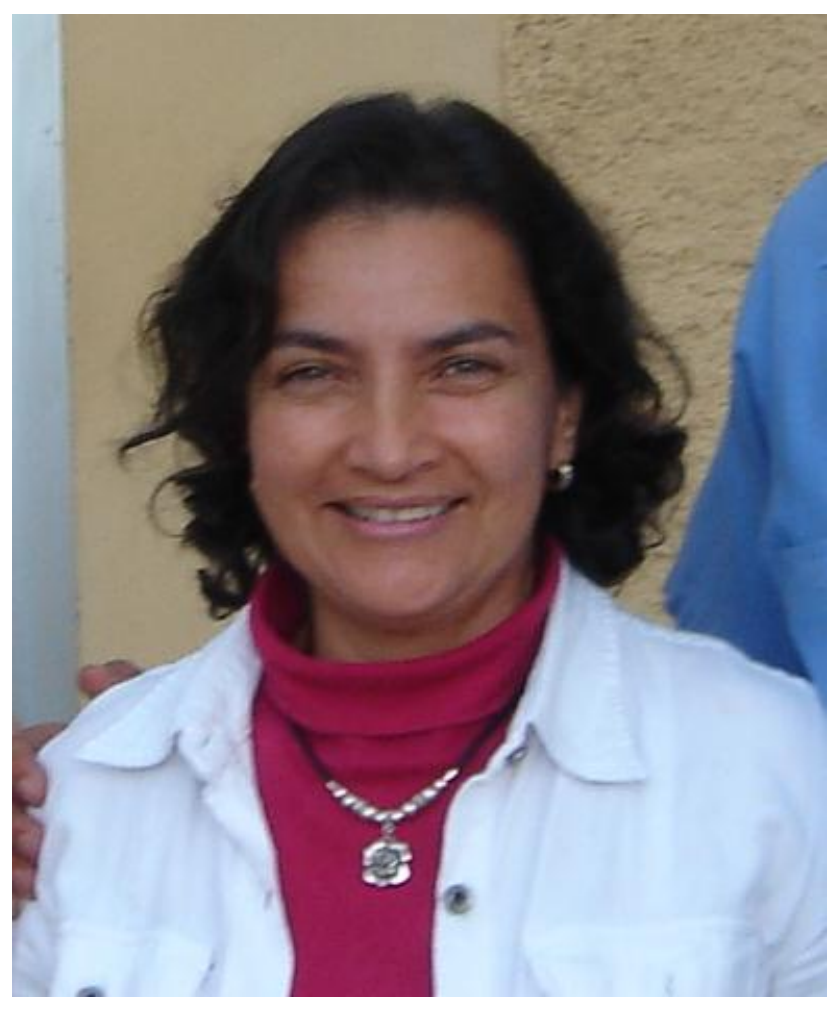

Foto 4. La Ing. Gloria Arévalo de Gauggel.

A partir de 2011, el curso básico de suelos es de cuatro créditos, lo que permite dar un curso más amplio e integral, incluyendo, además de los conceptos básicos de suelo, temas como el clima y su relación con el suelo, principios de nutrición vegetal y fuentes y calidad de agua para la agricultura, complementándolo con laboratorios prácticos que los estudiantes deben realizar cada semana, (Arévalo y Gauggel 2013), (Fotos 5 y 6). Los cursos avanzados son de tres créditos y de igual manera se realizan prácticas de laboratorio y campo cada semana (Arévalo y Gauggel 2011). En estos cursos, la academia se integra a la práctica, ya que los estudiantes desarrollan trabajos prácticos de campo en mapeo, ordenamiento y recomendaciones de manejo de suelos en el campus de Zamorano y en las cuencas de sus alrededores. Esta secuencia de trabajo ha permitido estudiar los suelos de Zamorano año a año, identificando cambios y necesidades de adaptar su manejo a las reales necesidades y actualizar la información. Con los resultados obtenidos, se diseñan medidas para evitar el deterioro del suelo, además que entrena al estudiante en técnicas de fácil y práctica aplicación, en su ejercicio profesional.

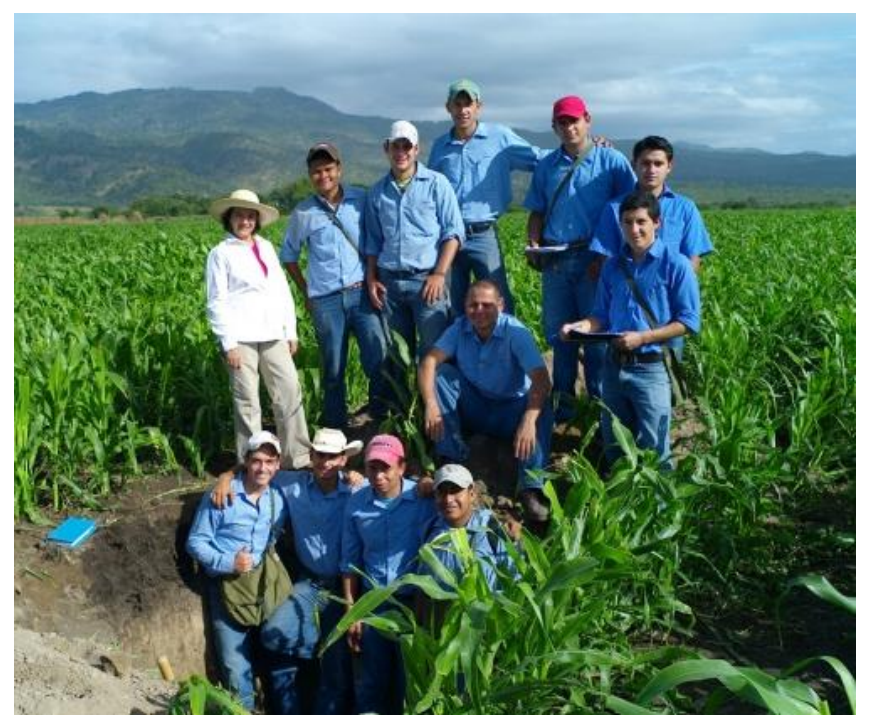

Foto 5. Calicata de suelos para su estudio realizada en los campos de Zamorano con estudiantes de primer y cuarto año en el 2009; a la izquierda la Ing. Gloria Arévalo de Gauggel.

En el 2005, se fundó el Club de Suelos de Zamorano por iniciativa de la estudiante Linda Estelí Méndez Barrientos (Clase 07), como actividad extracurricular con un enfoque académico y práctico. Este club ha sido bastante rico en participación de los estudiantes ya que desde su fundación mantiene en promedio 50 miembros cada trimestre y ha operado ininterrumpidamente desde su fundación. Se desarrollan actividades académicas con exposición de casos aplicados de la ciencia del suelo, con docentes de Zamorano, expositores externos y estudiantes de último año, donde exponen los resultados de su investigación desarrollada durante su pasantía o tesis. También hay actividades prácticas de campo que integran a los miembros más jóvenes al trabajo de campo de los cursos avanzados. Se han realizado dos giras internacionales, a Costa Rica en 2006 (Foto 7) y Guatemala en 2007 (Foto 8), donde se reconocieron suelos en su recorrido hacia estos países. 


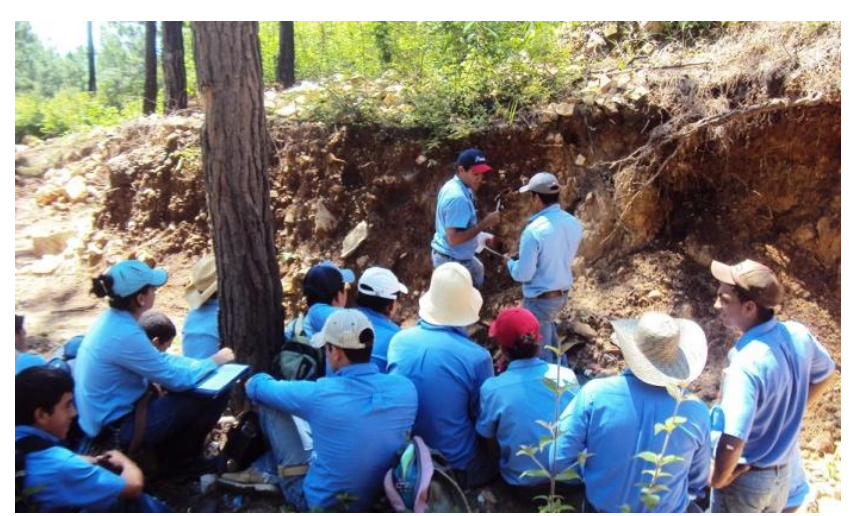

Foto 6. Práctica de campo en segundo año en el 2010.

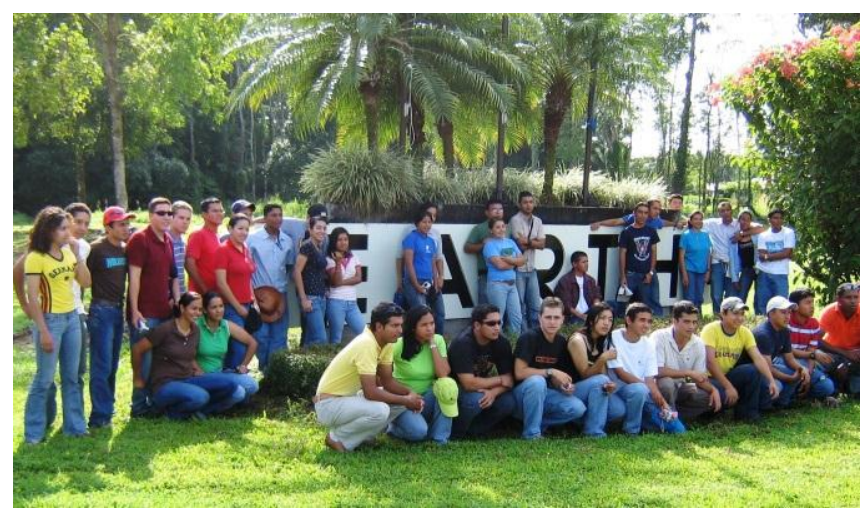

Foto 7. Gira del Club de Suelos de Zamorano a la Universidad EARTH en Costa Rica, octubre de 2006.

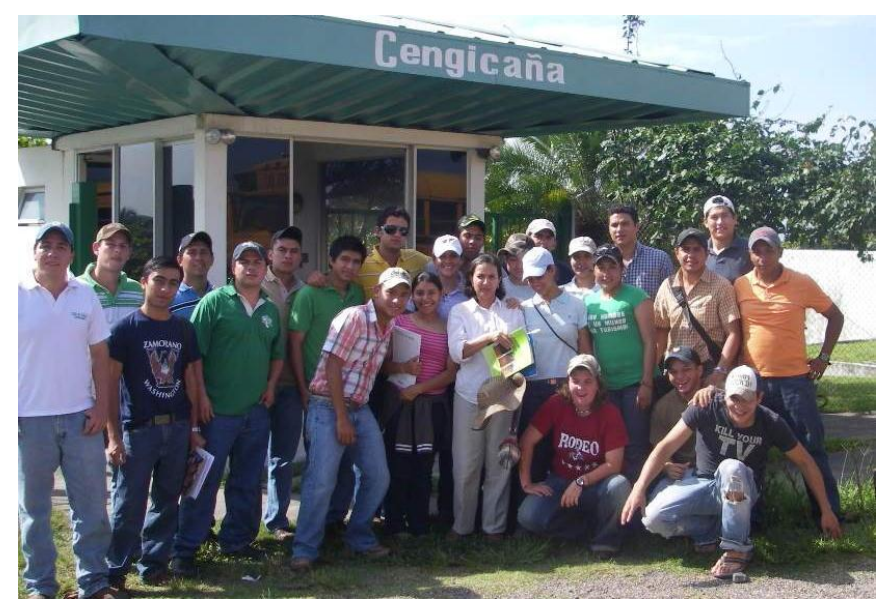

Foto 8. Gira del Club de Suelos de Zamorano a Guatemala, septiembre de 2007.
Con el cierre del Proyecto de Conservación de Suelos, PASOLAC, financiado por los suizos y por gestión del proyecto PROMIPAC en Honduras y su director el Dr. Alfredo Rueda, en el 2006 el proyecto pasó a Zamorano, convirtiéndose en el módulo de Manejo y Conservación de Suelos de Ladera, impartido en el aprender haciendo a los estudiantes de primer año (Fotos 9 y 10). El módulo inició como parte del programa de PROMIPAC y en el 2008 pasó a ser parte de la carrera de Ingeniería Agronómica. Desde el año 2010 el instructor de módulo es el Ing. Mario Raudes y se ha integrado el concepto de manejo del suelo con prácticas de conservación de suelos y producción intensiva de cultivos como hortalizas, musáceas, café y maíz. Este módulo permite capacitar al estudiante desde su primera experiencia en Zamorano, en el conocimiento y aplicación de conceptos básicos de manejo de suelos y en el diseño y aplicación de técnicas para la conservación de los suelos en zonas de ladera, tan comunes en toda Latinoamérica.

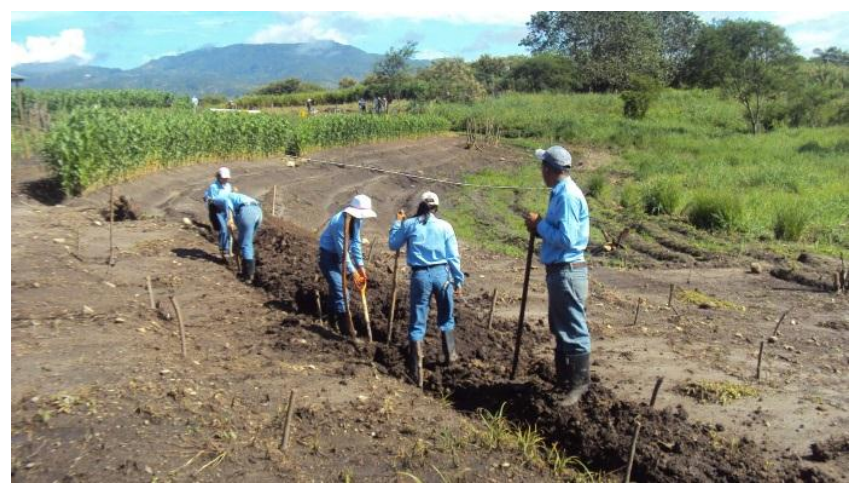

Foto 9. Actividad de los estudiantes de primer año en el módulo de suelos realizando obras de conservación de suelos en la parcela de laderas, Zamorano, 2010.

A través del programa de PROMIPAC, entre 2003 y 2012, se desarrollaron entrenamientos a técnicos y agricultores en Nicaragua, Honduras y El Salvador, logrando impactar ampliamente en el manejo de suelos de estos países. Con la Secretaría de Educación de Honduras se hicieron dos publicaciones para difundir conceptos básicos de manejo de suelos (Arévalo y Castellanos 2011, Raudes y Sagastume 2011). 


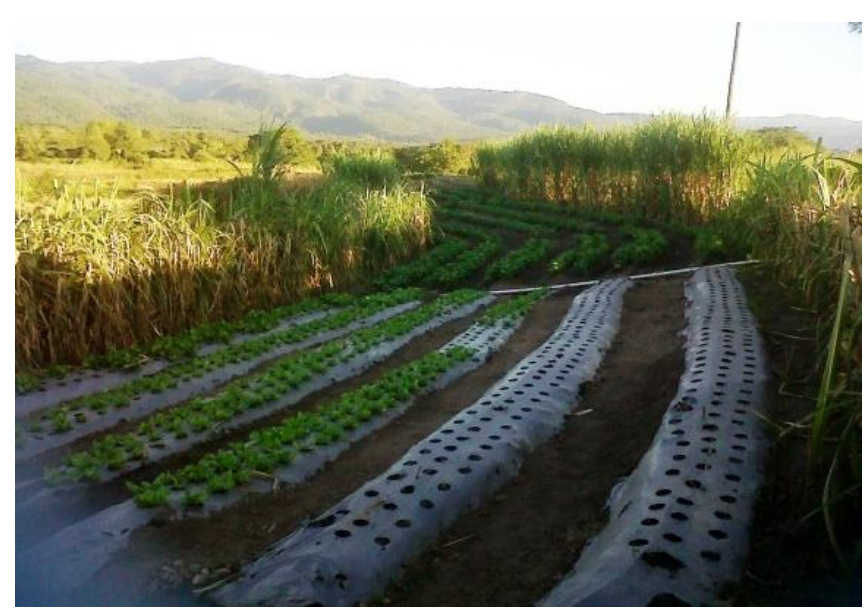

Foto 10. Vista de la parcela de conservación de suelos de Zamorano, 2012.

\section{Enfoque Docente del Programa de Suelos de Zamorano}

El programa de suelos está enfocado en la formación profesional de los estudiantes, en la comprensión e identificación de los procesos formadores de suelo y su aplicación práctica para su conservación y mejoramiento, a través de una explotación agrícola mejor y sostenible. Los tres cursos de suelos están dirigidos a incrementar los rendimientos de los cultivos de manera sostenible y a la conservación, rehabilitación y uso potencial del suelo, a través de la su clasificación. Se ha incursionado en temas de actualidad como el manejo de cultivos energéticos (Escobar Morán y Ronquillo Molina 2012) y también se han incorporado herramientas digitales en el mapeo de los suelos, a fin de tener información de fácil acceso y manejo de datos (Brito Mijares y Sarmiento Hernández 2012).

Las técnicas y los conceptos básicos de suelo se aplican en el manejo de los programas de producción y de suelos con los estudiantes. Las aplicaciones prácticas de los conceptos se practican en los laboratorios de las clases de suelos y con las tesis que han sido bastante numerosas y aplicadas. Los tesistas han colaborado con el trabajo de la unidad de suelos y de la parcela de conservación de suelos, de tal manera que el módulo y los laboratorios de campo son una actividad rutinaria (Fotos 11 y 12).

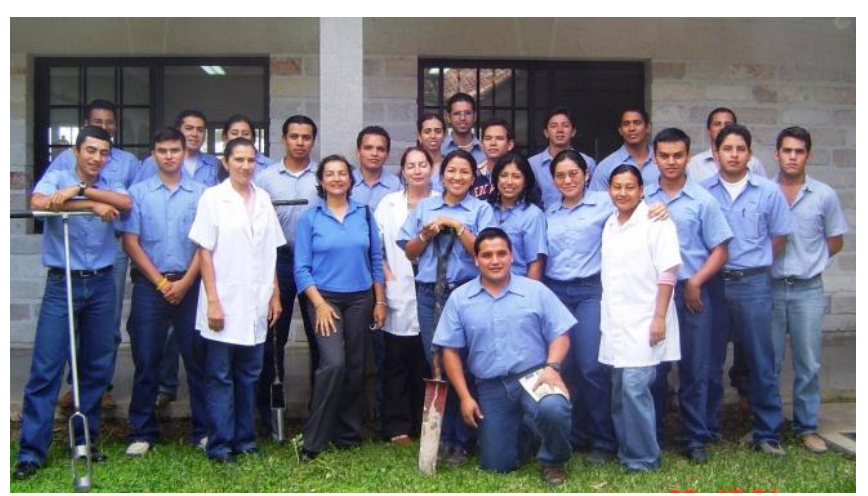

Foto 11. Tesistas de suelos de la clase 2005. Al centro Ing. Gloria Arévalo de Gauggel, de blanco la Ing. Hilda Flores (centro) y laboratoristas Martha Ávila (izquierda) y Jaqueline Moya (derecha).

En el 2002 y 2003 se realizó el mapa de suelos de las áreas de producción de Zamorano y en 2007 se amplió el estudio a todas las áreas, como parte del Plan Estratégico de Zamorano 2006-2010 (Chica et al. 2008, Dubón Fernández 2007, Velásquez et al. 2008). Los estudiantes participan en proyectos que la unidad de suelos lleva a cabo para Zamorano o para terceros, lo cual permite a los estudiantes hacer sus tesis en casos reales y de impacto en la producción agrícola. Los estudiantes son informados de los proyectos que la unidad de suelos y el laboratorio llevan a cabo.

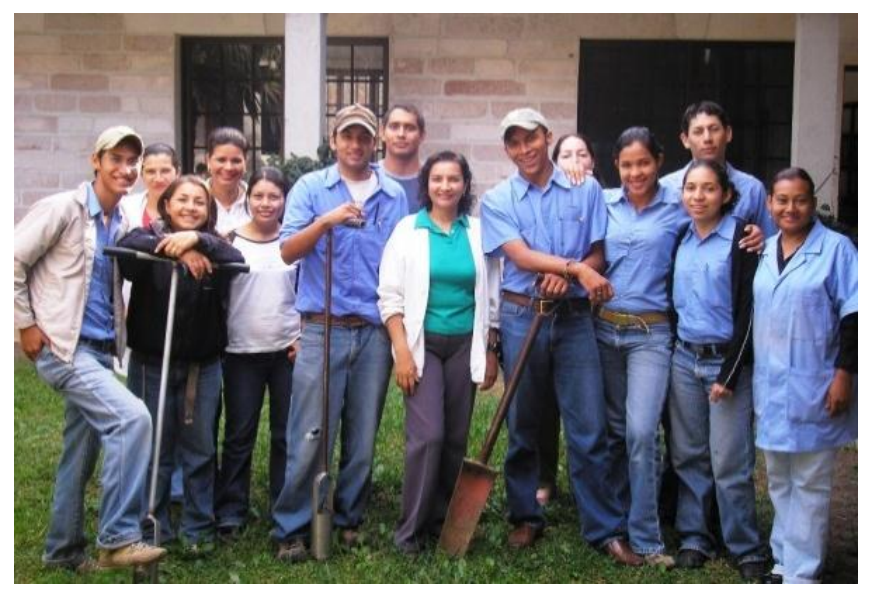

Foto 12. Tesistas de suelos de la clase 2006 y personal del Laboratorio de Suelos de Zamorano. 
Las tesis en temas de suelos y nutrición vegetal incrementaron sustancialmente desde el 2002, cuyos resúmenes están publicados en la revista Ceiba y algunos presentados en los congresos internacionales de suelos, en las áreas de fertilidad de suelos y nutrición vegetal se han realizado las tesis de Amador McCulloch y Bernal Chacón 2012, Baldeón Mendoza et al. 2009, Brenes et al. 2004, Cabrera García et al. 2009, Cárdenas et al. 2004, Castellanos Oseguera et al. 2008, Chavarría et al. 2008, Contreras Valenzuela et al. 2008, Durán Madrueña et al. 2008, Flores y Durán Aguirre 2008, Fúnez Oseguera et al. 2009, Gallo Dueñas et al. 2008, García et al. 2004, Garzón López 2006, Girón et al. 2004, Guamialamá Vela et al. 2008, Gurdián Torrez 2010, Mite Cáceres 2005, Navarrete Ganchozo 2005, Noruri Ferrufino et al. 2006, Padilla et al. 2008, Patiño Mejía 2005, Reyes et al. 2004, Santillán Núñez 2005, Saravia et al. 2004, Valeriano et al. 2004, Villavicencio Largacha 2005, y Zeledón et al. 2004.

El trabajo de la unidad de suelos se involucra con el de otras unidades como la de riego y drenaje y cultivos extensivos, frutales y horticultura, y también en la Carrera de Desarrollo Socioeconómico y Ambiente, en investigaciones relacionadas con los suelos (Aguilar Gudiel et al. 2009, Balladares Pesantes 2002, Banegas Cruz et al. 2009, Chicaiza et al. 2008, Hernández Carvajal 2002, Lemus et al. 2004, Marrone Del Cid et al. 2009, Maura Pazmiño 2002, Méndez Pineda et al. 2009, Molina Llano et al. 2009, Morales Cruz et al. 2009, Plaza et al. 2004, Reinoso Díaz et al. 2008, Sánchez Flores et al. 2008, Vinueza Iñiga et al. 2009, y Zambrano et al. 2004).

Algunos métodos analíticos del laboratorio han sido desarrollados con tesis (Oliva Escobar et al. 2009). La unidad de suelos ofrece como parte de sus servicios la resolución de problemas relacionados con el manejo de suelos, involucrándose en la resolución de problemas reales en la producción agrícola, en empresas de diferente magnitud de producción, en fertilidad de suelos y nutrición vegetal, adecuación y remediación de tierras, necesidades de drenaje, y estudios de suelos para la planificación agrícola.

La unidad de suelos ha aportado grandemente al establecimiento de procedimientos y aplicación ordenada del conocimiento a la producción agropecuaria en fincas o sistemas de producción, aumentando significativamente la producción. En estas actividades se involucran los estudiantes a través de proyectos de pasantías y tesis, formándolos para su futura gestión profesional en temas aplicados de manejo de suelos, conservación y cultivos (Baca Izaguirre et al. 2009, Castellanos Oseguera 2005, Cueva Manos 2003, Escoto Durón et al. 2009, López et al. 2004, Martínez Ponce et al. 2008, Mendoza Aguilar et al. 2008, Ochoa Burgos 2004, Orellana Bojorquez 2003, Reiche et al. 2012, Reyes et al. 2012, Rodriguez Márquez 2006, Rubiano Cárdenas 2010, Smith Miller 2012, Tipán Villareal 2006) y en la recuperación de la calidad del suelo (Bustos Estévez y Chicaiza Guishcaso 2011, Calderón et al. 2008, Galarza Brito 2011, Guerra Serrano y Mendieta Servellón 2011, y Pantoja Guamán 2005) y en el área de conservación de suelos se han desarrollado varios trabajos aplicados, contribuyendo de esta manera a entender y formular planes de protección contra la pérdida de suelo (Chávez Ramírez y Bojorquez Aguirre 2012, Esquivel Palma y Mendoza Barzola 2011, García Ramírez 2008, Gurdián Pacheco 2002, y Meléndez Rivera 2011).

En el 2011, y con el apoyo la contribución de los programas de PASOLAC y PROMIPAC, bajo la coordinación en Zamorano del Dr. Alfredo Rueda y en Coordinación con la Secretaria de Educación de Honduras, se publicó el libro Fertilizantes y Enmiendas (Arévalo y Castellanos 2011) y el de Conservación de Suelos (Raudes y Sagastume 2011). En 2012, con el mismo apoyo se capacitaron 200 técnicos del INTA en Nicaragua, como parte de la extensión en manejo de suelos.

Por contribuciones originales a la ciencia del suelo, la unidad de suelos ha recibido el reconocimiento como mejores trabajos de tesis en la carrera de Ingeniería Agronómica en el 2000, 2002 y 2009 (Barahona Flores 2000, Gurdián Pacheco 2002 y Oliva Escobar 2009) y en la carrera de Desarrollo Socioeconómico y Ambiente en 2012 (Acosta Velasquez y Kucharsky Lezama 2012). Los mejores trabajos de tesis se han presentado en los congresos Latinoamericanos de la Ciencia del Suelo, participando con científicos de todo el mundo y algunos de ellos han sido reconocidos por su calidad y aporte (Guardián Pacheco 2002, Oliva Escobar et al. 2009, Reiche et al. 2012, y Rivas et al. 2012). 
Se desarrolló un método de índices numéricos para calificación de la calidad de los suelos, identificando cuantitativamente las limitantes que tiene el suelo y su oportunidad de mejora (Gauggel et al. 2009). Ha aportado al conocimiento de los suelos de Honduras y la aplicación de los conceptos de suelos a la producción agrícola con resultados muy positivos en su mejoramiento $\mathrm{y}$ en el planeamiento $\mathrm{y}$ el ordenamiento de los recursos naturales (Rivas et al. 2012). La unidad de suelos de Zamorano se reconoce a nivel nacional y regional como un centro de conocimiento aplicado, unidad de servicio y de capacitación en la formación de los profesionales que egresan de Zamorano y también para la capacitación y actualización de profesionales agrícolas de diferentes instituciones y de agricultores en toda la región.

En el 2010, Zamorano, a través de sus docentes, fue invitado a participar en la ejecución del Atlas de Suelos de Latinoamérica, coordinado por el proyecto EUROCLIMA de los países Europeos, y en 2012 en el nuevo mapa de suelos del mundo coordinado por la FAO, representando a Honduras. A raíz de esta invitación, el Dr. Carlos Gauggel participó en la primera reunión de Rio en 2010 y en la reunión de la FAO en Roma en 2012, en el grupo de trabajo técnico Gestión del Suelo Vivo. La Ing. Arévalo ha participado en talleres en Argentina, Colombia y Brasil durante 2012. Este compromiso permitió integrar el trabajo con la carrera de Ambiente y Desarrollo y la Unidad de Sistemas de Información Geográfica y actualizar el mapa de suelos de Honduras, integrado al mapa de suelos de LatinoAmérica, consolidando la información de los suelos de Honduras registrada por la unidad de suelos a través de muchos años, mediante la tesis de Brito Mijares y Sarmiento Hernández (2012), usando el nuevo sistema mundial de clasificación de suelos WRB.

\section{Proyección de la Unidad de Suelos en la Actividad Agrícola Centroamericana}

Desde el 2002, la unidad de suelos ha colaborando y buscando oportunidades para apoyar al sector agroindustrial de la región. De esta manera, se han brindado servicios técnicos a varias empresas líderes de la industria cañera y producción de cultivos hortícolas, frutales y granos básicos, en empresas en
Honduras, Nicaragua y El Salvador, y con proyección a Ecuador, Panamá y Belice. Entre las empresas agrícolas a las que brinda servicio están los ingenios azucareros Compañía Azucarera Tres Valles, Azucarera La Grecia, Azucarera Choluteca, Ingenio Azucarero Chumbagua en Honduras, en Nicaragua a los ingenios azucareros de San Antonio y Monterosa, y a productores de café y hortalizas a través de los programas Fintrac, Placencia Tabaco, PMA Nicaragua, PMA Honduras, empresas que suministran fertilizante e insumos agrícolas y otras muchas, además de productores independientes.

La aplicación de los conceptos básicos de la ciencia del suelo aplicados a las recomendaciones de fertilización y prácticas como encalamiento, dirigidas a los usuarios del servicio de análisis de suelos, han resultado en beneficios para el agricultor, quien reconoce incrementos significativos en su producción al aplicar las recomendaciones que entrega el Laboratorio de Suelos junto con los resultados de los análisis. El impacto económico de estos resultados no se ha medido mediante un estudio, pero se reconoce que es millonario.

Los técnicos de muchas empresas e instituciones han recibido cursos básicos hasta avanzados en nutrición vegetal, fertilidad de suelos, interpretación de análisis de suelos y foliares, conservación y rehabilitación de suelos. Esta actividad es frecuentemente requerida y la unidad de suelos invierte esfuerzos sustanciales en capacitación de profesionales de instituciones privadas $y$ gubernamentales no solamente en Honduras, sino Centro América y el Caribe (República Dominicana).

Los programas de suelos generan más de $\$ 100,000$ al año que hacen autosostenible al laboratorio de suelos y al mismo tiempo cumple con su misión principal, la educación de excelente calidad y brindar el apoyo técnico al sector agrícola de varios países, convirtiéndose en institución de referencia en el área. El éxito en las recomendaciones de nutrición vegetal, y en general de manejo de suelos, ha generado una demanda significativa y creciente, en los servicios que el laboratorio ofrece.

En este momento, el laboratorio y los programas agrológicos trabajan a su capacidad máxima. La cartera de clientes del laboratorio incluye desde pequeños a grandes productores, ONG, y hasta el 
gobierno de Honduras. Existe un potencial muy grande para que los servicios de programas de suelos de Zamorano puedan expandirse a Ecuador, Panamá, Nicaragua y Bolivia para suplir las necesidades del sector agrícola. La capacitación que reciben los estudiantes en los cursos de suelos ha sido clave en la promoción y aplicación de conceptos de la ciencia del suelos para la protección de los suelos, generando programas integrales para su manejo. Los egresados Zamoranos son reconocidos por su conocimiento en el campo de suelos y tienen gran aceptación en el mercado laboral.

\section{Literatura Citada}

Acosta Velásquez, A.F., O.A Kucharsky Lezama. 2012. Estudio edafológico y de cobertura para la modelación hidrológica con el modelo SWAT de la microcuenca Santa Inés, Honduras. Tesis Ing. Desarrollo Socioeconómico y Ambiente, Zamorano, Honduras. 35 p.

Aguilar Gudiel J.C., N. Berger, G. Arévalo de Gauggel, L. Espinoza. 2009. Efecto del inhibidor NBPT en la volatilización de amoníaco proveniente de la hidrólisis de urea en un sistema semi estático y en pastos. Resumen. Ceiba 50(2):63.

Alpizar Vargas, N.F. s.f. Uso de lisímetros para evaluar el movimiento de Nitrógeno y Potasio en suelos bananeros de Coyoles, Honduras, con potencial de lixiviación. Tesis Ing. Agr., Zamorano, Honduras. 58 p.

Amador McCulloch, J.C., Bernal Chacón, I.E. 2012. Curva de absorción de nutrientes del cultivo de arroz (Oryza sativa L.) variedad Venezuela 21, en un suelo vertisol bajo condiciones del valle de Sébaco, Nicaragua. Proyecto Especial del Programa de Ing. Agr., Zamorano, Honduras. 32 p.

Andrade Yépez, L. 2003. Efecto del biofertilizante Mycoral en el crecimiento inicial de banano y de plátano en el campo de El Zamorano, Honduras. Tesis Ing. Agr., Zamorano, Honduras. 29 p.

Andrews, A.M., A. Castro, J.C. Rosas, F. Rodriguez. 1995. Respuestas de variedades de frijol a la fertilización fosforada en dos localidades de Honduras. Informe anual de Investigación. Departamento de Agronomía, EAP, Zamorano, IAI 7:90-91.

Andrews, A.M., A. Castro, J.C. Rosas., F. Rodriguez. 1994. Tolerancia del frijol a bajos niveles de fosfato. Informe anual presentado a ProFrijol. 13 p.
Andrews, A.M. 1993. Resumen de tesis 1993 del Departamento de Agronomía. Zamorano, Honduras. 20 p.

Andrews, A.M. 1990. Soil factors affecting bean (Phaseolus vulgaris L.) production in Honduras, Central America. Ph.D. Dissertation, Universidad de Florida, Gainesville, Florida. $234 \mathrm{p}$

Arévalo, G., M. Castellanos. 2011. Módulo 6. Fertilizantes y Enmiendas. Programa para la Agricultura Sostenible en Laderas de América Central. Carrera de Ciencia y Producción Agropecuaria. Escuela Agrícola Panamericana, Zamorano. Honduras. 59 p.

Arévalo, G.E., C.A. Gauggel. 2011. Manual de prácticas. Curso de Manejo de suelos y nutrición vegetal. CPA. Unidad de Suelos. Séptima edición. EAP Zamorano. 77 p.

Arévalo, G.E., C.A. Gauggel. 2013. Manual de laboratorio de ciencia de suelos y aguas. Segunda edición. Escuela Agrícola Panamericana Zamorano. Unidad de Suelos. Departamento de Ciencia y Producción Agropecuaria. $107 \mathrm{p}$.

Ávila Cáceres, I.D. 1991. Comportamiento de las plagas de brócoli (Brassica oleracea var. Itálica) bajo un sistema de producción orgánico. Tesis Ing. Agr., Zamorano, Honduras. 115p.

Baca Izaguirre, M.A., G. Arévalo de Gauggel, N. Berger, C. Gauggel, J.C. Quezada. 2009. Diseño de un sistema de drenaje y costo de implementación en cultivo de piñón, Choluteca Honduras. Resumen. Ceiba 50(2):65.

Balladares Pesantes, J.E. 2002. Comparación técnica y económica de cuatro niveles de fertilización con cuatro tamaños de bolsa en chile dulce bajo condiciones de macrotúnel en Zamorano. Tesis Ing. Agr. Zamorano, Honduras. 52 p.

Baldeón Mendoza, P.G., G. Arévalo de Gauggel, J. Pack, A. Rueda. 2009. Efecto de la aplicación de bio activado y silicio en la calidad del cultivo de alcachofa (Cynara scolymus L.) en Latacunga, Ecuador. Resumen. Ceiba 50(2):69.

Banegas Cruz, C.E., N. Berger, G. Arévalo de Gauggel, J. Leiva, J.C. Quezada. 2009. Influencia de la fertilización con N, P, K en el establecimiento en campo de Jatropha curcas L., en la finca Santa Lucía, Choluteca, Honduras. Resumen. Ceiba 50(2):54.

Barahona Flores, R., 2000. Caracterización detallada de los suelos de San Nicolás y prácticas recomendadas para su uso sostenible, El Zamorano, Honduras. Tesis Ing. Agr. Zamorano, Honduras. 51 p.

Barillas Lechuga, J.R. s.f. Impacto económico y agronómico de dos criterios de fertilización de tomate, pepino y maíz dulce bajo las condiciones de El Zamorano. Tesis Ing. Agr. Zamorano, Honduras. 54 p. 
Brenes, T., G. Gauggel, C. Gauggel, D. Moreira. 2004. Evaluación de biodisponibilidad de nutrientes bajo tres sistemas de riego y dos niveles de ácido húmico para caña de azúcar en el Valle de Cantarranas, Honduras. Resúmenes de investigaciones en Ciencias Agropecuarias. Vol II:34.

Brito Mijares, J.R., G.E. Sarmiento Hernández. 2012. Propuesta para la actualización del mapa de suelos de Honduras como base para diagnóstico de uso de suelo y contenido de carbón orgánico. Tesis Ing. Agr. Zamorano, Honduras. 43 p.

Bustos Estévez, C.A., C.A. Chicaiza Guishcaso. 2011. Evaluación del efecto del subsoleo del suelo en la producción de frijol Amadeus 77 y DEORHO en Zamorano, Honduras. Tesis Ing. Agr. Zamorano, Honduras. 25 p.

Cabanilla Burbano, E.V. 2005. Evaluación del efecto de Mycoral en el desarrollo de meristemos de banano en tres sustratos y dos dosis de fertilización en vivero en Honduras. Tesis Ing. Agr. Zamorano, Honduras. 13 p.

Cabrera García, J.C., G. Arévalo de Gauggel, J. Pack.2009. Evaluación del consumo de agua y nutrientes en chile (Capsicum annuum) variedades Taranto, Zidenka y Sympathy, en hidroponía y condiciones de altura en Mulacagua, Comayagua Honduras. Resumen. Ceiba 50(2):62.

Calderón A.P., G. Arévalo, M. Vélez, A. Rueda. 2008. Evaluación del efecto del subsoleo y tres niveles de fertilización en la producción del pasto estrella (Cynodon nlemfuensis) en Monteredondo, Zamorano, Honduras. Resumen. Ceiba 49(2):140.

Calderón Díaz, J.A. 2005. Evaluación del control de nematodos en el cultivo de Stevia con Micorriza VAM, en la E.A.P, Zamorano, Honduras. Tesis Ing. Agr. Zamorano, Honduras. 17 p.

Canales Paguada, J.S., G. Arévalo, O. Duarte, E.A. Cruz, E. Raddatz. 2008. Efecto de Mycoral ${ }^{\circledR}$ en vivero con dos niveles de fertilización en palma africana (Elaeis guineensis) en Atlántida, Honduras. Resumen. Ceiba 49(2): 188.

Cárdenas, C., G. Arévalo, J. Miselem, F. Arias. 2004. Determinación de los efectos en rendimiento de la producción de lechuga hidropónica y el convencional en condiciones de El Zamorano, Honduras. Resúmenes de investigaciones en Ciencias Agropecuarias. Vol. II: 61.

Castellanos Oseguera, L.A., G. Arévalo, J. Pack. 2008. Consumo de nutrientes y Evaluación del consumo de nutrientes y agua en chile (Capsicum annuum) variedades: Lírica, Simpathy y Zidenka, bajo condiciones de altura en Mulacagua, Comayagua, Honduras. Resumen. Ceiba 49 (2): 188.
Castellanos Oseguera, M.E. 2005. Efecto de la salud y calidad de suelos sobre la producción de plátano (Musa AAB) var. Curraré enano en la finca La Pita, Casa Quemada, San Pedro Sula, Honduras. Tesis Ing. Agr. Zamorano, Honduras. $49 \mathrm{p}$.

Castro Vargas, R.E. 2003. Caracterización detallada de los suelos de los sectores de Ganado Lechero y Monte Redondo 1, 2 y 3 del Zamorano, Honduras para el establecimiento y renovación de pasturas. Tesis Ing. Agr., Zamorano, Honduras. 34 p.

Chavarría, J., G. Arévalo, C. Gauggel, S. Pérez. 2008. Curvas de absorción de nutrientes en tabaco (Nicotiana tabacum), var. Habano Criollo bajo condiciones de manejo de campo en Jalapa, Nicaragua. Resumen. Ceiba 49(2):147.

Chávez Ramírez, K., J.E Bojorquez Aguirre. 2012. Plan de conservación de suelos en laderas y fertilización para el cultivo de café en Ahuachapán, El Salvador. Tesis Ing. Agr., Zamorano, Honduras. 26 p.

Chávez, D., A. Rueda, G. Arévalo de Gauggel, A. Jaco, R. Trabanino. 2006. Efecto de las Infecciones Virales en el Desarrollo del Cultivo de Chile Dulce (Capsicum annuиm L.) Inoculado con Micorrizas Vesículo Arbusculares (VAM) y Trichoderma harzianum. Resumen. Ceiba 47(1-2):-50.

Chica, R., G. Arévalo, C. Gauggel, M. Castellanos. 2008. Estudio general de suelos el sector de RAPACO, propiedad de la Escuela Agrícola Panamericana en Honduras y recomendaciones de prácticas para el uso sostenible. Resumen. Ceiba. 49(2):155.

Chicaiza, J., A. Rueda, G. Arévalo, D. Cedeño. 2008. Producción de lombriz roja californiana (Eisenia foetida) y lombrihumus con estiércol de vaca, cabra, cerdo y caballo. Resumen. Ceiba 49(2):147.

Coello Wilches, A.A. 2005. Efecto del Biofertilizante Mycoral en el crecimiento fisiológico de plátano con 5 meses de establecimiento en el campo de El Zamorano, Honduras. Tesis Ing. Agr., Zamorano, Honduras. 19p.

Contreras Valenzuela, A., C. de J. Gómez Vargas, G. Arévalo, J. Pack, A. Pitty. 2008. Evaluación de tres variedades de albahaca y dos dosis de fertilización en producción hidropónica y en suelo. Resumen. Ceiba 49(2): 198.

Crespo Mena, A.B. 2006. Efecto de micorrizas benéficas $\left(\right.$ Mycoral $\left.^{\circledR}\right)$ y cachaza, en el peso de la caña y rendimiento neto de azúcar, en la compañía Azucarera Tres Valles, Honduras Tesis Ing. Agr., Zamorano, Honduras. 31 p.

Cruz Ortiz, E.A. 2007. Efecto del Mycoral en Palma africana en vivero. Tesis Ing. Agr., Zamorano, Honduras. $16 \mathrm{p}$. 
Cueva Manos, F.F. 2003. Caracterización de suelos y fertilización de frutales de altura media alta en el Occidente de Honduras. Tesis Ing. Agr., Zamorano, Honduras. $21 \mathrm{p}$

De La Cadena Vera, J.E. 2005. Efecto de la aplicación de micorriza en rosas de corte bajo invernadero, en Inversiones Florícolas, Quito Ecuador. Tesis Ing. Agr., Zamorano, Honduras. 18 p.

Díaz, D. sf. Cuantificación de indicadores locales de calidad de suelo en la microcuenca de Luquigue, Yoro, Honduras. Tesis Ing. Agr., Zamorano, Honduras. 63 p.

Díaz Zelaya, J. 1989. Estudio de suelos a semidetalle del valle El Zamorano. Secretaría de Planificación, coordinación y presupuesto, Dirección ejecutiva del Catastro, Tegucigalpa, Honduras. 108 p.

Discua Duarte, S., O. Duarte., G. Arévalo, C. Martínez, M. Guachambala, E. Raddatz. 2008. Comparación entre cuatro envases para propagar café catuaí y Lempira por semilla con y sin inoculación con Mycoral. Resumen. Ceiba 49(2):191.

Dubón Fernández, E.J. 2007. Estudio general de suelos de las áreas de montaña en la Escuela Agrícola Panamericana, Zamorano, Honduras. Tesis Ing. Agr., Proyecto Especial del Programa de Ing. Agr., Zamorano, Honduras. 63 p.

Durán Madrueña, A., G. Arévalo, C. Gauggel, R. Solórzano, M. Castellanos. 2008. Determinación de curvas de absorción de nutrientes para la variedad Mex 79431 de caña de azúcar en la Compañía Azucarera Tres Valles, Cantarranas, Honduras. Resumen. Ceiba 49(2): 190.

Escobar Morán, J.J., O.E. Ronquillo Molina. 2012. Respuesta a la fertilización orgánica con el uso de Biol y potásica inorgánica en King grass (Pennisetum purpureum) para estimación energética de potencial productivo de biogás, Zamorano, Honduras. Proyecto Especial del Programa de Ing. Agr., Zamorano, Honduras. 27 p.

Esquivel Palma, C.J., C.Q. Mendoza Barzola. 2011. Plan de manejo y conservación de suelos para la producción de sandía y forraje en Zona 1, Escuela Agrícola Panamericana, Zamorano, Honduras. Proyecto Especial del Programa de Ing. Agr., Zamorano, Honduras. 40 p.

Erickson, N. 2001a. Manual de Conferencias. Ciencia de Suelos. Zamorano, Honduras. 98 p.

Erickson, N. 2001b. Manual de laboratorio. Ciencia de Suelos. Zamorano, Honduras. 64 p.

Escoto Durón, F.J., G. Arévalo de Gauggel, C. Gauggel, J. C. Quezada. 2009. Estudio semidetallado de suelos de la finca Santa Lucia, Choluteca, Honduras, para el cultivo de piñón (Jatropha curcas). Resumen. Ceiba 50(2):59.
Fernández Díaz, J.V. 2003. Caracterización detallada de los suelos de los sectores de Zorrales y Monte Redondo, de El Zamorano, Honduras para el establecimiento y renovación de pasturas. Proyecto Especial del Programa de Ing. Agr., Zamorano, Honduras. 44 p.

Flores, O.D., K. Durán Aguirre. 2008. Determinación de las curvas de absorción de nutrientes en la variedad CP722086 de caña de azúcar en los tres periodos de zafra 2007-2008 en el ingenio La Grecia, Choluteca, Honduras. Proyecto Especial del Programa de Ing. Agr., Zamorano, Honduras. 41 p.

Fúnez Oseguera, E.N., G. Arévalo de Gauggel, J. Pack, J. C. Quezada. 2009. Comparación de sustratos para la siembra de piñón (Jatropha curcas) en etapa de vivero, finca Santa Lucía, Choluteca, Honduras. Resumen. Ceiba 50(2):58.

Galarza Brito, P.J. 2011. Efecto del subsoleo y cultivo de cobertura (Dolichos lablab) en las propiedades físicas del suelo y producción de maíz cv. 30F32WHR, Zamorano, Honduras. Proyecto Especial del Programa de Ing. Agr., Zamorano, Honduras. 23 p.

Gallo Dueñas, T.S., G. Arévalo, W. Padilla, O. Duarte. 2008. El extracto celular de la inflorescencia de palma africana (Elaeis guineensis Jacq.), como herramienta de diagnóstico nutricional. Resumen. Ceiba 49(2):203.

García, C., G. Arévalo, C. Gauggel, D. Moreira. 2004. Evaluación agronómica y económica de tres programas de fertilización en caña de azúcar, Compañía Azucarera Tres Valles, Valle de Cantarranas, Honduras. Resúmenes de investigaciones en Ciencias Agropecuarias Volumen II:35.

García Ramírez, R.F. 2008. Evaluación de siete coberturas para el control de erosión en el cultivo de piña, bajo las condiciones de Montecristo, Departamento de Atlántida, Honduras. Proyecto Especial del Programa de Ing. Agr., Zamorano, Honduras. 12 p.

Garzón López, S.S. 2006. Evaluación del rendimiento de tres variedades de lechuga bajo el sistema NFT (nutrient film technique) de hidroponía con dos soluciones de nutrientes. Proyecto Especial del Programa de Ing. Agr., Zamorano, Honduras 26 p.

Gauggel, C., G. Arévalo., R. Barahona. 2009. Índices de calidad de suelos para las propiedades morfológicas, físicas y químicas. Memorias del XVIII Congreso Latinoamericano de la Ciencia del Suelo y VI Congreso Nacional de Suelos. Asociación Costarricense de la Ciencia del Suelo. p 52.

Girón, G., G. Arévalo, O. Duarte, E. Gurdián, R. Barahona. 2004. Caracterización química de los suelos de una finca de piña en Río Cuarto de Grecia, Costa Rica, para mejorar su condición nutricional. Resúmenes de investigaciones en Ciencias Agropecuarias Volumen II:36. 
Guamialamá Vela, E.H., G. Arévalo, A. Rueda, A. Sierra. 2008. Evaluación de la salud del cultivo de chile orgánico (Capscium annuum L.) usando ácido salicílico con gallinaza, bocashi o lombrihumus. Resumen. Ceiba 49(2):194.

Guerra González, J.E., G. Arévalo de Gauggel, J.C. Rosas, E. Cruz. 2009. Determinación del efecto de micorriza seleccionada cepa 4 en semillero y vivero de café variedad caturra. Resumen. Ceiba 50(2):57.

Guerra Serrano, A.J., J.A. Mendieta Servellón. 2011. Subsoleo en suelos arcillosos masivos y fertilización con magnesio en el cultivo de pasto Tobiatá (Panicum maximum). Proyecto Especial del Programa de Ing. Agr., Zamorano, Honduras. 27 p.

Gurdián Pacheco, E. 2002. Efecto de los acondicionadores de suelo y coberturas en el control de erosión en un cultivo intensivo mecanizado en la zona Atlántica de Costa Rica. Proyecto Especial del Programa de Ing. Agr., Zamorano, Honduras. 48 p.

Gurdián Torrez, J.L. 2010. Validación de una sonda de lixiviación como método para determinar la fertilidad del suelo en el cultivo de café (Coffea arabica), en la Escuela Agrícola Panamericana (EAP), Honduras Proyecto Especial del Programa de Ing. Agr., Zamorano, Honduras. 25 p.

Hernández Carvajal, J.I. 2002. Comparación costo-beneficio entre sistemas productivos y su relación con el estado nutricional de los suelos en tres microdrenajes en la microcuenca El Zapotillo. Proyecto Especial del Programa de Ing. Agr., Zamorano, Honduras. 89 p.

Lagos Molina, S.M. 2010. Evaluación de la efectividad de cinco cepas de micorriza benéfica, Zamorano, Honduras. Proyecto Especial del Programa de Ing. Agr., Zamorano, Honduras. 18 p.

Lavaire Cruz, T., E.E. Fiallos. 2010 Potencial de captura de agua de los suelos de la microcuenca El Carrizal, Parque Nacional La Tigra, Francisco Morazán, Honduras. Proyecto Especial del Programa de Ingenieros en Desarrollo Socioeconómico y Ambiente, Zamorano, Honduras. 48 p.

Lemus, S., P. Paz, G. Arévalo. 2004. Efecto de tres coberturas y dos sistemas de labranza sobre maíz y frijol bajo tres niveles de fertilización. Resúmenes de investigaciones en Ciencias Agropecuarias Volumen II:37.

Lin, S.Y. 1954. Informe del Dr. S.Y. Lin. FAO Fishieries Officer. Secretaría de Estado, en el despacho de Agricultura, República de Honduras. Pg. 8. Mecanografiado. November 1954.
Lizama Meza, P.A., J.A. Vásquez Guillén. 2001. Evaluación biológica y económica del uso de micorrizas (Mycoral) en cuatro pastos. Proyecto Especial del Programa de Ing. Agr., Zamorano, Honduras. 29 p.

López, A., G. Arévalo, C. Gauggel, E. Gurdián. 2004. Características del suelo en el cultivo del maíz bajo labranza cero y labranza convencional en suelos típicos del Valle de Quimistán, Honduras. Resúmenes de investigaciones en Ciencias Agropecuarias Volumen II:38.

López Aguilar, J. 2003. Caracterización de suelos de la Zamoempresa de Cultivos Extensivos en el Valle de El Zamorano, Honduras. Proyecto Especial del Programa de Ing. Agr., Zamorano, Honduras. 35 p.

Malo, S.E. 1999. El Zamorano: Afrontando el reto de la América tropical. Impreso en Cuenca Ecuador, Sud América por Imprenta Monsalve Moreno y Cia. Ltda. p 179, 191.

Marrone Del Cid, J.A., N. Berger, G. Arévalo, J.N. Leiva. 2009. Efecto de las deficiencias nutricionales en el cultivo de piñón (Jatropha curcas). Resumen. Ceiba 50(2):60.

Martínez Martínez, C.M. 2008. Potencial de captura de agua en los suelos de Capiro y Zapotillo, Güinope, El Paraíso, Honduras. Proyecto Especial del Programa de Ingeniera en Desarrollo Socioeconómico y Ambiente, Zamorano, Honduras. 39 p.

Martínez Ponce, C.H., G. Arévalo, F. Alvarez, R. Solórzano. 2008. Evaluación de cuatro tiempos de suspensión del riego previo a la cosecha de la caña de azúcar variedad CB 3822, en el Ingenio Tres Valles, Cantarranas, Honduras. Resumen. Ceiba 49(2):200.

Maura Pazmiño, G. 2002. Determinación y valoración económica de la erosión hídrica en terrenos afectados por incendios forestales en Tatumbla, Departamento de Francisco Morazán, Honduras. Especial del Programa de Ing. en Desarrollo Socioeconómico y Ambiente, Zamorano, Honduras. 65 p.

Meléndez Rivera, V.B. 2011. Evaluación de la pérdida de suelo por erosión en el cultivo de macadamia (Macadamia integrifolia), en la finca Mocá Grande, Suchitepéquez, Guatemala. Proyecto Especial del Programa de Ing. en Desarrollo Socioeconómico y Ambiente, Zamorano, Honduras. 32 p.

Méndez Pineda, J.C., C.A. Chacón Tábora, J. Pack, G. Arévalo de Gauggel, U. Barahona. 2009. Evaluación del abono orgánico y sintético en la producción de zapallo (Cucurbita pepo). Resumen. Ceiba 50(2):70. 
Mendoza Aguilar, E. J., F. Alvarez, G. Arévalo, N. Berger. 2008. Diseño y cálculo de un sistema de drenaje en la finca San Nicolás, El Zamorano, Honduras. Resumen. Ceiba 49(2):194.

Mite Cáceres, J.R. 2005. Curva de absorción de nutrientes del cultivo de caña de azúcar en el valle de Cantarranas, compañía azucarera Tres Valles, Francisco Morazán, Honduras. Proyecto Especial del Programa de Ing. Agr., Zamorano, Honduras. 40 p.

Molina Llano, D., F. Alvarez, G. Arévalo de Gauggel, A. Fonseca. 2009. Evaluación del efecto productivo y económico de dos métodos de riego en Crisantemos (Chrysantehemum morifolium) en macro túnel, en la finca Los Silos, Antioquia, Colombia. Resumen. Ceiba 50(2):56.

Monroe, B., Jr. 1968. A distributional survey of the birds of Honduras. Ornithological Monographs. Allen Press Inc. Kansas USA. P15.

Morales Cruz, N.R., J. Pack, G. Arévalo de Gauggel, U. Barahona, D. Pu. 2009. Comparación de seis sustratos comunes en la producción de pepino (Cucumis sativa) y acumulación de sales, bajo invernadero en Zamorano, Honduras. Resumen. Ceiba 50(2):64.

Morales Rivera, C.A. 2005. Efecto del Mycoral en interacción con dos niveles de fertilización y de fósforo en producción de caña de azúcar en la Compañía Azucarera Tres Valles, Honduras. Proyecto Especial del Programa de Ing. Agr., Zamorano, Honduras. 30 p.

Navarrete Ganchozo, R.J. 2005. Curvas de absorción de nutrientes en el cultivo de pepino (Cucumis sativus L.) bajo condiciones de campo en Zamorano. Proyecto Especial del Programa de Ing. Agr., Zamorano, Honduras. $21 \mathrm{p}$.

Noruri Ferrufino, G. Arévalo de Gauggel, H. Flores, U. Barahona. 2006. Determinación de la Concentración de la Solución Nutritiva Para Crecimiento y Producción de Lechuga var. Verónica en Hidroponía. Resumen. Ceiba 47(1-2):51-67.

Nuñez Servellón, N. s.f. Caracterización física y química de suelos manejados bajo gramíneas forrajeras y cultivos extensivos en El Zamorano, Honduras. Proyecto Especial del Programa de Ing. Agr., Zamorano, Honduras. 35 p.

Ochoa Burgos, J.P. 2004. Evaluación de índices de calidad de suelos cultivados con banano en fincas comerciales típicas del Valle de Sula, Honduras. Proyecto Especial del Programa de Ing. Agr., Zamorano, Honduras. 36 p.
Oliva Escobar, D.P., G. Arévalo de Gauggel, C. Gauggel, H.E. Flores, A. Rueda. 2009. Determinación de la acidez intercambiable $\left(\mathrm{Al}^{+3}+\mathrm{H}^{+}\right)$a partir del $\mathrm{pH}$ para la estimación de la capacidad de intercambio catiónico (CIC) en suelos de la cuenca del Pacífico en El Salvador, Honduras y Nicaragua. Resumen. Ceiba 50(2):55.

Orellana Bojorquez, S.V. 2003. Caracterización de suelos y fertilización de frutales de clima templado en el occidente de Honduras. Proyecto Especial del Programa de Ing. Agr., Zamorano, Honduras. 25 p.

Padilla, A., G. Arévalo, C. Gauggel. 2008. Curvas de absorción de nutrientes de la rosa variedad Rockefeller bajo condiciones de macrotúnel en la empresa Agroganadera Espinoza Chiriboga, Cotopaxi, Ecuador. Resumen. Ceiba 49(2):141.

Paniagua, A., A.M. Andrews, M. Avedillo, J. Kammerbauer. 1998. Relationship of Soil Characteristic to Vegetation Successions on a Sequence of Degraded and Rehabilitated Soils in Honduras, Tegucigalpa, Honduras: Elsevier- P.IRR.

Pantoja Guamán, J.L., 2005. Efecto del subsoleo en las propiedades físicas y químicas del suelo y en el rendimiento de cuatro cultivos en Zamorano, Honduras. Proyecto Especial del Programa de Ing. Agr., Zamorano, Honduras. 79 p.

Patiño Mejía, L. 2005. Evaluación de hierro y manganeso en la eliminación de puntos necróticos en crisantemo (Dendrathema grandiflorum Kytamura) variedad Yellow Vero en Antioquia, Colombia. Proyecto Especial del Programa de Ing. Agr., Zamorano, Honduras. $26 \mathrm{p}$.

Patiño Mejía, R.A. 2005. Evaluación del uso de micorrizas y dos niveles de fertilización en producción de crisantemo (Dendrathema grandiflorum Kytamura) en Zamorano, Honduras. Proyecto Especial del Programa de Ing. Agr., Zamorano, Honduras. 26 p.

Pérez, J. 1996. Influencia del fósforo en el comportamiento agronómico de cuatro genotipos de soya. El Zamorano, Honduras. Proyecto Especial del Programa de Ing. Agr., Zamorano, Honduras. 45 p.

Plaza, F., M. Vélez, G. Arévalo, F. Alvarez. 2004. Determinación del requerimiento hídrico de cuatro pastos mediante el uso de lisímetros bajo condiciones de El Zamorano, Honduras. Resúmenes de investigaciones en Ciencias Agropecuarias Vol. II:20. 
Posada, L., P. Paz, G. Arévalo, B. Reyes. 2004. Efecto de tres niveles de fósforo sobre la asociación de Mycoral $^{\circledR}$ con tres genotipos de maíz. Resúmenes de investigaciones en Ciencias Agropecuarias. Vol.II:45.

Pozo Gordillo, B. s.f. Efecto del uso de obras de conservación de suelos sobre los rendimientos de sorgo y frijol y características del suelo en Namasigue y El Ocotal, Honduras. Proyecto Especial del Programa de Ing. Agr., Zamorano, Honduras. 44 p.

Raudes, M., N. Sagastume. 2011. Conservación de Suelos. Módulo 3. Programa para la agricultura sostenible en laderas de América Central. Carrera de Ciencia y Producción Agropecuaria. Escuela Agrícola Panamericana, El Zamorano. Honduras. 75 p.

Reiche, A., G. Arévalo, C. Gauggel, C. Morales, D. Oliva. 2012. Fijación de fósforo y potasio en dos suelos del Valle de Cantarranas, Honduras. En: Memorias de XIX Congreso Latinoamericano de la Ciencia del Suelo y XXIII Congreso Argentino de la Ciencia del Suelo. Mar del Plata, Argentina. 6 p.

Reilly, J.P., P. Trutmann, A. Rueda, D.E. Matute L, S. López, R. Izaguirre, R. Dominguez y A. Pitty 2002. Guía de Salud de Suelos. Manual para el cuidado de la salud de suelos: Universidad de Cornell y Zamorano. Grupo de trabajo sobre suelos, Tegucigalpa, Honduras. Reilly J, Trutmann P., Rueda A. y Grupo de Salud de suelos (eds).160 p.'

Reinoso Díaz, A.J., P.A. Ruiz Ruiz, J. Pack, G. Arévalo de Gauggel. 2008 Determinación de la mejor cantidad de agua y relaciones carbono: nitrógeno para el establecimiento de una compostera. Resumen. Ceiba 49(2):216.

Reyes, J.M., G. Arévalo., C. Gauggel., H. Pocasangre. 2012. Estudio de la evolución pedogenética post Mitch en los suelos de la zona bananera, Olanchito, Departamento de Yoro, Honduras. En: Memorias de XIX Congreso Latinoamericano de la Ciencia del Suelo y XXIII Congreso Argentino de la Ciencia del Suelo. Mar del Plata, Argentina. 6 p.

Reyes, V., C. Gauggel, G. Arévalo. 2004 Determinación de índices de biodisponibilidad de nutrientes por tres métodos analíticos bajo dos condiciones de manejo de suelo para sorgo en Zamorano, Francisco Morazán, Honduras. Resúmenes de investigaciones en Ciencias Agropecuarias. Vol. II: 41.

Rivas, D., C. Proaño, G. Arévalo, M. Cortés. 2012. Rehabilitación de suelos por contaminación con hidrocarburos mediante bioremediación. En: Memorias de XIX Congreso Latinoamericano de la Ciencia del Suelo y XXIII Congreso Argentino de la Ciencia del Suelo. Mar del Plata, Argentina. 6 p.
Rodriguez Márquez, G.G. 2006. Evaluación de la condición de drenaje y propuesta de mejoramiento de la vega del Río Yeguare. EAP Zamorano, Honduras. Proyecto Especial del Programa de Ing. Agr., Zamorano, Honduras. $38 \mathrm{p}$.

Romero Oseguera, G.A. 2006. Evaluación del efecto de la Micorrizas VAM en el cultivo de café, El Paraíso, Honduras. Proyecto Especial del Programa de Ing. Agr., Zamorano, Honduras. 24 p.

Rubiano Cárdenas, M.I. 2010. Estudio exploratorio de suelos en el municipio de Ojojona, Francisco Morazán, Honduras. Proyecto Especial del Programa de Ing. en Desarrollo Socioeconómico y Ambiente, Zamorano, Honduras. $41 \mathrm{p}$.

Sánchez Flores, R.A., M. Vélez, G. Arévalo, I. Matamoros. 2008. Efecto del encalado superficial sobre la producción de los pastos estrella (Cynodon nlemfuensis) y transvala (Digitaria eriantha) en suelos moderadamente ácidos de Monterredondo II, Zamorano, Honduras. Resumen. Ceiba 49(2):186.

Santillán Núñez, V.N. 2005. Efecto de la aplicación de Metalosato de calcio y magnesio en la producción de Rosa spp. variedades Charlotte y Classy bajo invernadero en Pichincha, Ecuador. Proyecto Especial del Programa de Ing. Agr., Zamorano, Honduras. 28 p.

Saravia, F., J. Miselem, G. Arévalo, C. Gauggel. 2004. Elaboración de curvas de absorción de nutrientes para la variedad de tomate (Lycopersicon esculentum Mill) Alboran bajo condiciones de invernadero en Zamorano, Honduras. Resúmenes de investigaciones en Ciencias Agropecuarias Vol. II:63.

Segura Escobar, M.B. 2010. Efecto de N, P, K y Mycoral $^{\circledR}$ sobre el desarrollo vegetativo en el primer año de Jatropha curcas L. var. Cabo Verde, Zamorano, Honduras. Proyecto Especial del Programa de Ing. Agr., Zamorano, Honduras. 33 p.

Smith Miller, A. M. 2012. Diseño de un sistema de evacuación de agua superficial para Zorrales, Zamorano, Honduras. Proyecto Especial del Programa de Ing. Agr., Zamorano, Honduras. 32 p.

Tamashiro, J. 1997. Absorción de nitrógeno y fósforo en maíz (Zea mays L.) y frijol (Phaseolus vulgaris L.) bajo condiciones del Zamorano. EAP, Tegucigalpa, Honduras. 44 p.

Terrones Cano, C.A. 2003. Caracterización edáfica de las áreas cultivadas con frutales en Zamorano, Honduras. Proyecto Especial del Programa de Ing. Agr., Zamorano, Honduras. 50 p. 
Tipán Villarreal, M.R. 2006. Estudio detallado de suelo de la Finca Inversiones Florícola S. de R.L, Checa, Pichincha, Ecuador. Proyecto Especial del Programa de Ing. Agr., Zamorano, Honduras. 48 p.

Urrutia Tobar, C.M. 2000. Efecto del uso de obras de conservación del suelo y el rendimiento de maíz y sorgo en el sur de Honduras. Proyecto Especial del Programa de Ing. Agr., Zamorano, Honduras. 59 p.

Valeriano, M., G. Arévalo, P. Paz, C. Gauggel, E. Gurdián. 2004. Desarrollo de un proceso para rehabilitar suelos arcillosos dedicados al cultivo de arroz por inundación, a la producción de plátano. Resúmenes de investigaciones en Ciencias Agropecuarias Volumen II:42.

Vásquez Maldonado, M.A. 1994. Efecto de la incorporación de frijol, abono, compost y estiércol sobre algunas características del suelo y el rendimiento del maíz y frijol común. Proyecto Especial del Programa de Ing. Agr., Zamorano, Honduras. 77 p.

Velásquez, D., G. Arévalo, C. Gauggel, S. Damas. 2008. Estudio semidetallado de suelos de la parte plana de la Escuela Agrícola Panamericana, Zamorano, Honduras. Resumen. Ceiba 49(2):143.

Villavicencio Largacha, C.X. 2005. Comparación de tres programas de nutrición en tomate (Lycopersicon esculentum) bajo invernadero en Zamorano, Honduras. Proyecto Especial del Programa de Ing. Agr., Zamorano, Honduras. 23 p.
Vinueza Iñiga, R.J., F. Alvarez, N. Berger, G. Arévalo de Gauggel. 2009. Diseño de un plan de manejo del riego para los cultivos de cítricos en el Zamorano, Honduras. Resumen. Ceiba 50(2):68.

Wong C., I.A. 1992. Efecto de la fertilización con nitrógeno y fósforo sobre la fijación de nitrógeno y rendimiento en frijol común. Proyecto Especial del Programa de Ing. Agr., Zamorano, Honduras. 73 p.

Yuksel, H., O. Robles. 1979. Informe técnico del estudio semidetalle de suelos en el valle de Zamorano. Programa de Catastro Nacional, Departamento de Recursos Naturales, Sección de Suelos. Informe provisional.

Zambrano, G., F. Alvarez, M. Vélez, G. Arévalo. 2004. Evaluación de tres coeficientes del cultivo $(\mathrm{Kc})$ para determinar necesidades de riego en Panicum maximum cv Tobiatá bajo condiciones de El Zamorano, Honduras. Resúmenes de Investigaciones en Ciencias Agropecuarias Vol. II:23.

Zeledón, S., G. Arévalo, J. Miselem. 2004. Evaluación del manejo de nutrientes y agua para el cultivo de tomate (Lycopersicon esculentum Mill) bajo condiciones de macrotúnel en Zamorano, Honduras. Resúmenes de investigaciones en Ciencias Agropecuarias Vol. II:64.

Recibido para publicación el 31 de julio de 2012. Aceptado para publicación el 2 de junio de 2013. 